\title{
Improvement of Mott spin polarimeters: an overlooked redundancy
}

Mauro Fanciulli ${ }^{1,2,3, a)}$

${ }^{1)}$ Laboratoire de Physique des Matériaux et des Surfaces, CY Cergy Paris Université, 95031 Cergy-Pontoise, France

2) Université Paris-Saclay, CEA, CNRS, LIDYL, 91191 Gif-sur-Yvette, France

3) Institut de Physique, École Polytechnique Fédérale de Lausanne, 1015 Lausanne, Switzerland

(Dated: January 31, 2020)

In Mott polarimeters the measurement of the spin polarization of an electron beam along two perpendicular quantization axes is commonly performed by collecting scattered electrons with four independent detectors placed azimuthally at $90^{\circ}$ from each other. However, the four intensities are not independent quantities. This overlooked redundancy will be pointed out, and it will be shown how to use it in order to better design, calibrate and operate Mott polarimeters.

\footnotetext{
a) mauro.fanciulli@u-cergy.fr
} 


\section{INTRODUCTION}

A Mott polarimeter is a device extensively used since more than sixty years in many different fields such as atomic, molecular, solid state, nuclear and high energy physics ${ }^{1}$, that allows to measure the spin polarization $\boldsymbol{P}$ of an electron beam. The vector $\boldsymbol{P}$ is an ensemble property of the beam, where each spatial components describes the normalized difference of electrons with spin up $N^{\uparrow}$ and spin down $N^{\downarrow}$ along that quantization axis: $P_{i}=\left(N_{i}^{\uparrow}-N_{i}^{\downarrow}\right) /\left(N_{i}^{\uparrow}+N_{i}^{\downarrow}\right)$ with $i=x, y, z$. The measurement of the spin polarization of an electron beam is important in many scientific applications, as for example in spin- and angle-resolved photoelectron spectroscopy (SARPES) ${ }^{2,3}$. However, it is generally a difficult task because all the existing spin polarimeters are rather inefficient devices. Despite the drawback of having a particularly low figure of merit and thus being highly time consuming, Mott polarimeters are still a reliable choice especially for quantitative spin analysis. In fact, they probe the two spin channels of a certain spin quantization axis in parallel, instead of relying on sequential measurements as in other kinds of spin polarimeters, where some parameter is changed such as magnetization of the magnetic target in very low energy electron diffraction (VLEED) polarimeters ${ }^{4,5}$, or target orientation and incident energy in spin mirror polarimeters ${ }^{6}$. Another method that allows parallel detection is the spin-polarized low energy electron diffraction (SPLEED) polarimeter ${ }^{7,8}$. By taking into account the diffraction geometry of a SPLEED polarimeter it will be possible to find an analogous redundancy to the one pointed out in this publication for Mott polarimeters.

An important issue for quantitative spin analysis in parallel detection is the calibration of the different detectors involved, in order to avoid the introduction of an experimental spin asymmetry. A Mott polarimeter consists of a heavy element target, usually a gold foil, onto which the electron beam is sent perpendicularly with high energy of a few decades of $\mathrm{keV}^{9-11}$. The sensitivity to the spin polarization is due to the spin-dependent relativistic scattering of electrons by a central potential, known as Mott scattering ${ }^{12}$. A standard configuration of a Mott polarimeter consists of four detectors placed azimuthally at $90^{\circ}$ from each other around the scatterer. As it will be explained later, this allows to determine the two spatial components of $\boldsymbol{P}$ in the plane of the scatterer. A second polarimeter can be placed at $90^{\circ}$ from the first one, allowing to access the third spatial component.

In this publication it will be shown that the four intensities measured in each polarimeter 
are not independent quantities. To the best of my knowledge, this redundancy has never been explicitly described in the literature nor is used in common practice, while it has several useful consequences in terms of design, calibration and operation of a Mott polarimeter. In order to illustrate this redundancy, it is convenient to first summarize the description of the Mott scattering process. By treating it with the relativistic Dirac equation, one can find the expression of the differential scattering cross-section $\frac{d \sigma_{\text {tot }}}{d \Omega} \doteq \sigma^{13}$ :

$$
\sigma(\theta, \phi)=I(\theta)[1+S(\theta) \boldsymbol{P} \cdot \boldsymbol{n}(\phi)]
$$

where the angles $\theta$ and $\phi$ describing the polar and azimuthal directions of the scattered electron beam are shown in Fig. 1(a), $I$ is the electron total intensity, and $S$ is the socalled Sherman function ${ }^{14}$, that is a given real function of the complex scattering amplitude parameters. The direction $\boldsymbol{n}$ is the unitary vector perpendicular to the scattering plane defined by the electron momenta before and after scattering, $\hbar \boldsymbol{k}$ and $\hbar \boldsymbol{k}^{\prime}: \boldsymbol{n}= \pm \frac{\boldsymbol{k} \times \boldsymbol{k}^{\prime}}{\left|\boldsymbol{k} \times \boldsymbol{k}^{\prime}\right|}$, where the positive sign is for forward scattering and negative sign for backscattering, that is the case considered in Fig. 1(a) and normally used in Mott polarimeters. The azimuthal $\phi$ angle is defined with respect to the red axis in Fig. 1(a), which corresponds to the direction of the transverse component $P_{t}$ of the spin polarization. Eq. (1) can be rewritten as:

$$
\sigma(\theta, \phi)=I(\theta)\left[1-S(\theta) P_{t} \sin \phi\right]
$$

as it can be checked by trigonometric inspection. In this way, the asymmetry $A_{b a}$ of the number of electrons $N$ scattered towards two different azimuthal positions $b$ and $a$ identified by the angles $\phi_{b}$ and $\phi_{a}$ is given by

$$
A_{b a}=\frac{N_{b}-N_{a}}{N_{b}+N_{a}}=\frac{\sigma_{b}-\sigma_{a}}{\sigma_{b}+\sigma_{a}}=P_{t} S \sin \phi_{a}
$$

where $a$ and $b$ have been chosen so that $\phi_{b}=2 \pi-\phi_{a}$. From Eq. (3) it follows that by measuring $A$ by placing two detectors around the target at azimuthal directions $a$ and $b$ it is possible to obtain $P_{t}$. In particular, choosing $\phi_{a}=90^{\circ}$ one has the very well-known equation of the standard Mott polarimeter: $A=P_{t} S$. From this it can be seen why the Sherman function is often considered as the spin-resolving power of the polarimeter, since in this particular configuration it determines the amount of asymmetry observed for a fully polarized beam. 
In the following, although a new proposal of multichannel detection of electrons with an angular distribution in a Mott polarimeter exists ${ }^{15}$, the standard single channel Mott polarimeter still commonly used will be considered for simplicity. Also, the particular SARPES experimental setup at the COmplete PHotoemission Experiment (COPHEE) endstation ${ }^{16}$ of the SIS beamline at the Swiss Light Source will be considered as an example. However, the results can be easily adapted to any particular geometry, and eventually to the multichannel case.

\section{THE REDUNDANCY IN STANDARD MOTT POLARIMETERS}

The geometry of the 3D Mott polarimeter installed at COPHEE is shown in Fig. 1(b), where the electron beam is alternatively sent every second into one of the two polarimeters called $L$ and $R$ and placed at $90^{\circ}$. Each polarimeter has four detectors placed at azimuthal directions of $90^{\circ}$ from each other. In this way, the asymmetries corresponding to two perpendicular spin quantization axes are measured with couples of detectors that are at opposite scattering directions $\left(\phi_{a}=90^{\circ}\right)$, which maximizes Eq. (3) as seen before. Since one Mott polarimeter can give information only about the spin polarization in the plane of the scattering target, the use of a second polarimeter allows to access also the third spatial component. In Fig. 1(c) the numbering of the eight diodes used as detectors at COPHEE is shown.

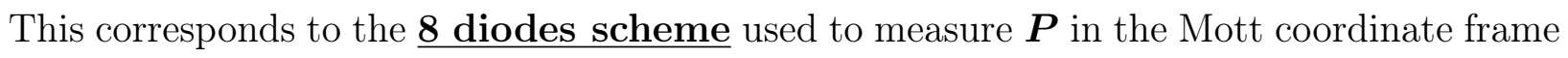
$(x, y, z)$. For example, if the component $P_{t}$ to be measured is chosen along $y$ one has $b$ and a corresponding to the diodes $L_{4}$ and $L_{2}$ respectively [see Fig. 1(c)]. The set of equations used to measure $\boldsymbol{P}$ is the following:

$$
\begin{gathered}
P_{i}^{\kappa}=\frac{1}{S} A_{i}^{\kappa}, \\
A_{y}^{L}=\frac{L_{4}-L_{2}}{L_{4}+L_{2}}, \\
A_{z}^{L}=\frac{L_{3}-L_{1}}{L_{3}+L_{1}}, \\
A_{x}^{R}=\frac{R_{4}-R_{2}}{R_{4}+R_{2}}, \\
A_{z}^{R}=-\frac{R_{3}-R_{1}}{R_{3}+R_{1}}=A_{z}^{L},
\end{gathered}
$$




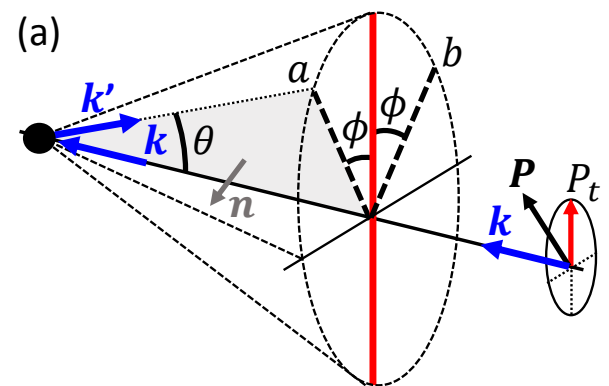

(c)

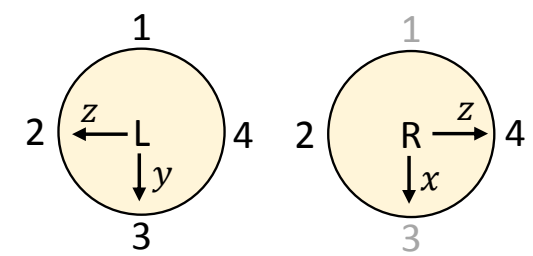

(b)

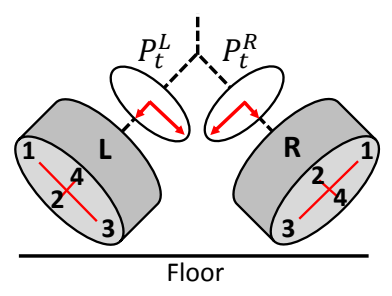

(d)

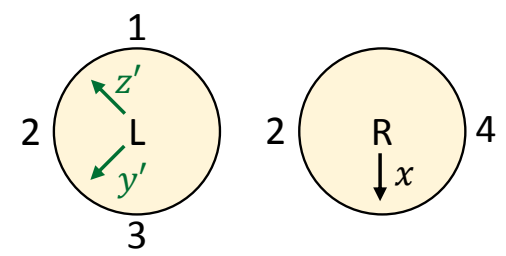

Figure 1. (a) Geometry of the Mott scattering. (b) The labeling of the detectors used in the two Mott polarimeters at COPHEE. View from the back of the hemispherical energy analyzer. (c) The 8 diodes scheme. The 6 diodes scheme is without $R_{1}$ and $R_{3}$, in grey. (d) The 5 diodes scheme.

where $\kappa=L, R$ and $i=x, y, z$ as shown in Fig. 1(c). Measuring the eight electron intensities $L_{1-4}$ and $R_{1-4}$ one obtaines all the spin asymmetries. Even if the 8 diodes scheme is the most commonly used in practice, the asymmetry for the $z$ component is clearly redundant, as shown in Eq. (8), since it is measured in both $L$ and $R$ polarimeters. Whereas in practice $A_{z}$ is recorded as the average of the two separate measurements, in principle it is possible to consider only the $\underline{\mathbf{6} \text { diodes scheme }}$ as the standard of three-dimensional spin polarization measurements. However, there is one more redundancy which is much less obvious. The

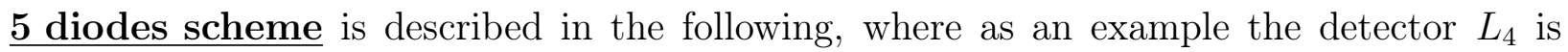
considered to be the redundant one.

Keeping the very same experimental geometry but now considering $\phi_{a}=45^{\circ}$ instead of $90^{\circ}$, it is possible to define two new axis $y^{\prime}$ and $z^{\prime}$ as shown in Fig. 1(d). Now the asymmetry equations are the following:

$$
\begin{aligned}
& A_{y^{\prime}}^{L}=\frac{L_{3}-L_{2}}{L_{3}+L_{2}}=P_{y^{\prime}} S \frac{1}{\sqrt{2}}, \\
& A_{z^{\prime}}^{L}=\frac{L_{2}-L_{1}}{L_{2}+L_{1}}=P_{z^{\prime}} S \frac{1}{\sqrt{2}},
\end{aligned}
$$

where the last term is obtained from Eq. (3). By combining Eq. (3),(5),(9),(10) and by 
considering that $P_{y}=\frac{1}{\sqrt{2}}\left(P_{y^{\prime}}-P_{z^{\prime}}\right)$ one can write:

$$
P_{y}=\frac{1}{S} A_{y}=\frac{1}{S} \frac{L_{4}-L_{2}}{L_{4}+L_{2}}=\frac{1}{\sqrt{2}}\left(P_{y^{\prime}}-P_{z^{\prime}}\right)=\frac{1}{\sqrt{2}} \frac{\sqrt{2}}{S}\left(\frac{L_{3}-L_{2}}{L_{3}+L_{2}}-\frac{L_{2}-L_{1}}{L_{2}+L_{1}}\right)
$$

from which it follows:

$$
\frac{L_{4}-L_{2}}{L_{4}+L_{2}}=\frac{L_{3}-L_{2}}{L_{3}+L_{2}}-\frac{L_{2}-L_{1}}{L_{2}+L_{1}} .
$$

By solving Eq. (12) with respect to $L_{4}$ one thus obtains:

$$
L_{4}^{*}=L_{2} \frac{L_{1} L_{2}-L_{2} L_{2}+3 L_{1} L_{3}+L_{2} L_{3}}{L_{1} L_{2}+3 L_{2} L_{2}-L_{1} L_{3}+L_{2} L_{3}}
$$

which shows the redundancy of the four detectors. The star symbol is used to highlight the fact that the intensity in diode 4 is not the measured one $L_{4}$, but is analytically obtained from Eq. (13). However, analytically one has $L_{4}^{*}=L_{4}$. Now Eqs. (4)-(7) can be used with $L_{4}^{*}$ instead of $L_{4}$ in order to obtain the asymmetries. A similar calculation can be done for all the other diodes, depending on which one is chosen to be the redundant one ${ }^{17}$.

The fact that only three out of four detectors in a Mott polarimeter are independent (or five out of six for three dimensional spin measurements) as expressed by Eq. (13) has several implications:

1. It can be used as a guideline for calibration. In fact, a limiting factor for a good functioning of a Mott polarimeter is the necessity of adjusting the sensitivity of the four diodes so to not introduce an experimental asymmetry. In standard practice, this is achieved by regularly performing reference measurements. Instead, by using Eq. (13) and equivalent equations for the other detectors ${ }^{17}$ it is possible to recursively calibrate the four diodes. For example, if one finds $L_{4}^{*} \neq L_{4}$ then the new calibration method is to modify the sensitivity of the diodes by trial and error or via an appropriate algorithm until $L_{4}^{*}=L_{4}$.

2. It can be exploited as an additional way to routinely check data consistency and the correct operation of the apparatus during an experiment, in addition to the common redundancy of the 8 diodes with respect to 6 .

3. It allows for different designs of a Mott polarimeter. For example, a highly symmetric design would be made by only three diodes placed at $120^{\circ}$ from each other in each polarimeter. On the other hand, in special situations where the design is limited by 
hindrance the three diodes could be spaced by only a small angle. The evaluation of the uncertainty in each case will be different, along the lines of what is presented in the next sections.

4. It can be extremely useful in practice, in the case of one of the diodes being damaged in a conventional configuration. This is in particular a powerful quick fix in the short term in case of failure of one diode during an experiment.

5. It can be exploited in order to judge and eventually correct data measured in a conventional configuration with a posteriori data processing. In fact, each asymmetry can be obtained not only from a couple of opposite detectors, but also from different sets of three detectors considered separately.

\section{ANALYSIS OF UNCERTAINTIES IN THE 5 DIODES SCHEME}

It is useful to evaluate the uncertainty on the measurement of the spin polarization in the case of the 5 diodes scheme. It is well known for Mott polarimeters ${ }^{18}$ that, assuming Poissonian statistics for the intensity $L_{a, b}$ in the diodes $a$ and $b$, the uncertainty on the intesity is given by:

$$
\Delta L_{a, b}=\sqrt{L_{a, b}} .
$$

Assuming a not varying Sherman function is then easy to evaluate ${ }^{18}$ the uncertainty on the asymmetry $A_{b a}=\left(L_{b}-L_{a}\right) /\left(L_{b}+L_{a}\right)$ :

$$
\Delta A_{a b}=A_{a b} \sqrt{\left(\frac{\Delta\left(L_{b}-L_{a}\right)}{L_{b}-L_{a}}\right)^{2}+\left(\frac{\Delta\left(L_{b}+L_{a}\right)}{L_{b}+L_{a}}\right)^{2}}=\sqrt{\frac{1}{L_{b}+L_{a}}+\frac{\left(L_{b}-L_{a}\right)^{2}}{\left(L_{b}+L_{a}\right)^{3}}} \approx \sqrt{\frac{1}{L_{b}+L_{a}}},
$$

where the approximation is valid for sufficiently large $L_{a}$.

Now, since from Eq. (12) $A_{y}^{*}=A_{y^{\prime}}-A_{z^{\prime}}$, one can calculate the uncertainty on $A_{y}^{*}$ as:

$$
\Delta A_{y}^{*}=\sqrt{\left(\Delta A_{y^{\prime}}\right)^{2}+\left(\Delta A_{z^{\prime}}\right)^{2}}=\sqrt{\frac{1}{L_{3}+L_{2}}+\frac{1}{L_{2}+L_{1}}+\frac{\left(L_{3}-L_{2}\right)^{2}}{\left(L_{3}+L_{2}\right)^{3}}+\frac{\left(L_{2}-L_{1}\right)^{2}}{\left(L_{2}+L_{1}\right)^{3}}} \approx \sqrt{\frac{1}{L_{3}+L_{2}}+\frac{1}{L_{2}+L_{1}}} .
$$

The uncertainty on the measurement of $P_{y}$ using the 5 diodes scheme is then given by $\Delta P_{y}^{*}=\Delta A_{y}^{*} / S$. It is interesting to compare this uncertainty with the one for $P_{y}$ measured directly from intensities $L_{4}$ and $L_{2}$, that is $\Delta P_{y}=\frac{1}{S \sqrt{L_{4}+L_{2}}}$ :

$$
\frac{\Delta P_{y}^{*}}{\Delta P_{y}}=\sqrt{\frac{\left(L_{1}+2 L_{2}+L_{3}\right)\left(L_{2}+L_{4}\right)}{\left(L_{2}+L_{3}\right)\left(L_{2}+L_{1}\right)}} \approx \sqrt{2}
$$


by considering $L_{1} \approx L_{2} \approx L_{3} \approx L_{4}$. The factor $\sqrt{2}$ can be also found when directly calculating the uncertainty on $P_{y^{\prime}}: \Delta P_{y^{\prime}}=\frac{\sqrt{2}}{S \sqrt{L_{3}+L_{2}}}$, which follows from Eqs. (9) and (15). In combination with Eq. (3) it is interesting to note that, more in general, the correction factor is $1 / \sin \phi_{a}$. Concerning the other spatial component $P_{z}$, the evaluation of the uncertainty is the same since $A_{z}^{*}=-A_{y^{\prime}}-A_{z^{\prime}}$, and therefore one has:

$$
\Delta P_{z}^{*}=\Delta P_{y}^{*} \approx \frac{1}{S} \sqrt{\frac{1}{L_{3}+L_{2}}+\frac{1}{L_{2}+L_{1}}} .
$$

This approach does not explicitly take into account the strong non-linearity of Eq. (13). A better approach consists in evaluating the uncertainty on the intensity $L_{4}^{*}$ from Eq. (13), rewritten as $L_{4}^{*}=L_{2} \frac{N}{D}$, and then directly calculating the uncertainty on $A_{y}^{*}=\frac{L_{4}^{*}-L_{2}}{L_{4}^{*}+L_{2}}$. Rather tedious calculations give the following result:

$$
\begin{gathered}
\Delta N=\sqrt{L_{1} L_{2}\left(L_{1}+L_{2}\right)+2 L_{2}^{3}+9 L_{1} L_{3}\left(L_{1}+L_{3}\right)+L_{2} L_{3}\left(L_{2}+L_{3}\right)}, \\
\Delta D=\sqrt{L_{1} L_{2}\left(L_{1}+L_{2}\right)+18 L_{2}^{3}+L_{1} L_{3}\left(L_{1}+L_{3}\right)+L_{2} L_{3}\left(L_{2}+L_{3}\right)} \\
\Delta L_{4}^{*}=L_{2} \frac{N}{D} \sqrt{\frac{(\Delta N)^{2}}{N^{2}}+\frac{(\Delta D)^{2}}{D^{2}}+\frac{1}{L_{2}}} \\
\Delta A_{y}^{*}=\sqrt{\frac{2\left(N^{2}+D^{2}\right)}{L_{2} D^{2}(N+D)^{4}}\left(D^{4}+N^{2} D^{2}+L_{2}\left(D^{2}(\Delta N)^{2}+N^{2}(\Delta D)^{2}\right)\right)}
\end{gathered}
$$

It is found that $\Delta A_{y}^{*}$ from Eq. (22) is numerically almost identical to the one from Eq. (16) for sufficiently large and similar values of $L_{1}, L_{2}, L_{3}$, while it deviates from it and eventually diverges only for a small value of one of the three intensity. This shows how the use of the 5 diodes scheme is reliable only when the intensity on the diodes is sufficiently large.

To summarize this section, it is found that the use of the 5 diodes schemes instead of 6 leads to an uncertainty that is a factor of $\sqrt{2}$ larger. It is clear that the uncertainty will be worse, since the total counts in 5 diodes are less than in 6 . However it can still be acceptable in case of failure of one of the diodes. Instead, if a special design with different value of $\phi_{a}$ is considered, the uncertainty will be a factor of $1 / \sin \phi_{a}$ larger.

\section{DISCUSSION AND CONCLUSION}

In Fig. 2 an example to illustrate the redundancy is shown. In the top panel of (a) four raw momentum distribution curves (MDCs) measured with detectors $L_{1}, L_{2}, L_{3}$ and $L_{4}$ at 

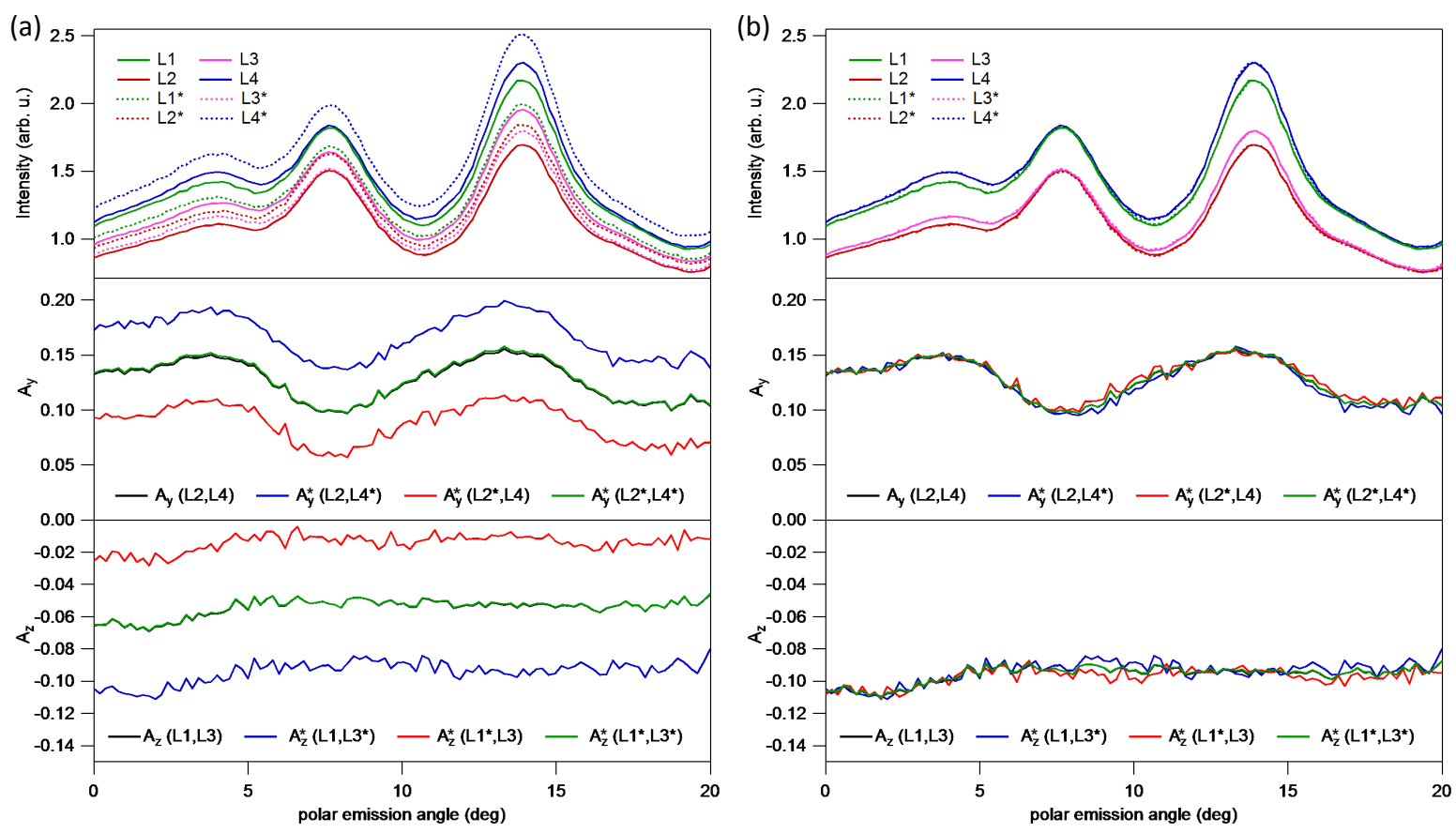

Figure 2. (a) Top: raw MDCs on $\mathrm{Bi} / \mathrm{Ag}(111)$ surface alloy ${ }^{19}$ measured with $L_{1}, L_{2}, L_{3}$ and $L_{4}$ (full line). The reconstructed $L_{1}^{*}, L_{2}^{*}, L_{3}^{*}$ and $L_{4}^{*}$ are shown as dotted line. Middle: asymmetries $A_{y}$ evaluated with all combinations of $L_{2}, L_{4}, L_{2}^{*}$ and $L_{4}^{*}$. Bottom: asymmetries $A_{z}$ evaluated with all combinations of $L_{1}, L_{3}, L_{1}^{*}$ and $L_{3}^{*}$. (b) Same as in (a), where $L_{3}$ has been multiplied by 0.92 .

COPHEE are shown. The MDCs were measured on $\mathrm{Bi} / \mathrm{Ag}(111)$ surface alloy ${ }^{19}$. The reconstruction of $L_{1}^{*}, L_{2}^{*}, L_{3}^{*}$ and $L_{4}^{*}$ from Eq. (13) and equivalent ones ${ }^{17}$ are also shown. Despite Eq. (13) being analytically exact, its strong non-linearity might result in a bad reconstruction if the standard previous calibration of the diodes was not correct. Indeed, in the case of Fig. 2 the reconstructed curves are not identical to the measured one. Nevertheless, the resulting asymmetry $A_{y}^{*}$ simply differ from $A_{y}$ by a constant, as shown in the middle panel of Fig. 2 (a). Here the quantity $A_{y}^{*}$ is evaluated in three different ways for completeness: $A_{y}^{*}=\frac{L_{4}^{*}-L_{2}}{L_{4}^{*}+L_{2}}, A_{y}^{*}=\frac{L_{4}-L_{2}^{*}}{L_{4}+L_{2}^{*}}$ and $A_{y}^{*}=\frac{L_{4}^{*}-L_{2}^{*}}{L_{4}^{*}+L_{2}^{*}}$. Similar result is found for $A_{z}^{*}$, as shown in the bottom panel. In worse situations where non-linear technical issues might compromise even further the quality of the calibration, the resulting asymmetries can also additionally differ by a multiplication factor. However, several tests run on different data sets always show at least the same qualitative trend for the asymmetries.

This observation points out a way to judge the limitations of quantitative analysis of a particular spin measurement. The example of Fig. 2 (a) is a data set that is quantitatively 
reliable because the asymmetry curves $A_{y}$ and $A_{y}^{*}$ differ only by a constant, as well as $A_{z}$ and $A_{z}^{*}$. That is to say, only the position of the zero of asymmetry has to be properly determined. This is reflected in the common practice of setting the actual position of the zero in the asymmetry curve by using some kind of reference as an additional input during standard data analysis (by setting the zero where it should be physically zero, e.g. far from a peak). Instead, the use of the redundancy pointed out in this paper allows for a different approach that is self-referenced, possibly as a live tool during the actual calibration or at least with a posteriori data treatment. For example, the intensities $L_{1-4}$ of Fig. 2 (a) have been slightly modified by trial and error until the reconstructed curves $L_{1-4}^{*}$ and asymmetries $A_{y, z}^{*}$ all match with $L_{1-4}$ and $A_{y, z}$ respectively. The result is shown in Fig. 2 (b), which is obtained in this case by multiplying the raw curve of only $L_{3}$ by a factor of 0.92 . This mimicks a better calibration of that diode, since all the curves now match extremely well. Better routines than trial and error can be implemented, in order to have control on the four intensities at the same time and to find the best situation where all curves match, and ideally the asymmetries values are automatically zero where they should physically be zero.

To conclude, in this paper a redundancy overlooked in the literature between the four detector intensities in a standard Mott polarimeter has been pointed out. It is of broad interest to different communities where electron spin measurements are involved, since it has several implications in terms of design, calibration and operation of a Mott polarimeter as well as data analysis.

\section{ACKNOWLEDGMENTS}

The author is very grateful to J. H. Dil, K. Hricovini and V. Petrov for their encouragement, valuable comments and useful discussions. This work was supported by the Swiss National Science Foundation Project No. P2ELP2_181877.

\section{REFERENCES}

${ }^{1}$ T. J. Gay and F. B. Dunning, "Mott electron polarimetry," Rev. Sci. Instrum. 63 (1992). 
${ }^{2}$ T. Okuda, "Recent trends in spin-resolved photoelectron spectroscopy," Journal of Physics: Condensed Matter 29, 483001 (2017).

${ }^{3}$ J. H. Dil, "Spin- and angle-resolved photoemission on topological materials," Electronic Structure 1, 23001 (2019).

${ }^{4}$ R. Bertacco and F. Ciccacci, "Oxygen-induced enhancement of the spin-dependent effects in electron spectroscopies of Fe(001)," Phys. Rev. B 59, 4207-4210 (1999).

${ }^{5}$ M. Escher, N. B. Weber, M. Merkel, L. Plucinski, and C. M. Schneider, "FERRUM: A New Highly Efficient Spin Detector for Electron Spectroscopy," e-Journal of Surface Science and Nanotechnology 9, 340-343 (2011).

${ }^{6}$ C. Tusche, M. Ellguth, A. A. Ünal, C.-T. Chiang, A. Winkelmann, A. Krasyuk, M. Hahn, G. Schönhense, and J. Kirschner, "Spin resolved photoelectron microscopy using a twodimensional spin-polarizing electron mirror," Applied Physics Letters 99, 32505 (2011).

${ }^{7}$ J. Kirschner and R. Feder, "Spin Polarization in Double Diffraction of Low-Energy Electrons from W(001): Experiment and Theory," Phys. Rev. Lett. 42, 1008-1011 (1979).

${ }^{8}$ D. Yu, C. Math, M. Meier, M. Escher, G. Rangelov, and M. Donath, "Characterisation and application of a SPLEED-based spin polarisation analyser," Surface Science 601, 5803-5808 (2007).

${ }^{9}$ V. N. Petrov, V. V. Grebenshikov, B. D. Grachev, and A. S. Kamochkin, "New compact classical 40 kV Mott polarimeter," Review of Scientific Instruments 74, 1278-1281 (2003).

${ }^{10}$ V. N. Petrov, M. Landolt, M. S. Galaktionov, and B. V. Yushenkov, "A new compact $60 \mathrm{kV}$ Mott polarimeter for spin polarized electron spectroscopy," Review of Scientific Instruments 68, 4385-4389 (1997).

${ }^{11}$ V. N. Petrov, M. S. Galaktionov, B. V. Yushenkov, Y. A. Mamaev, and M. Landolt, "Novel compact 60-kV Mott detector for spin-polarization electron spectroscopy," Technical Physics 43, 991-996 (1998).

${ }^{12}$ N. F. Mott, "The Scattering of Fast Electrons by Atomic Nuclei," Proceedings of the Royal Society of London. Series A 124, 425-442 (1929).

${ }^{13}$ J. Kessler, Polarized Electrons, 2nd edition (Springer, Berlin Heidelberg, 1985).

${ }^{14}$ N. Sherman, "Coulomb Scattering of Relativistic Electrons by Point Nuclei," Phys. Rev. 103, 1601-1607 (1956).

${ }^{15}$ V. N. Strocov, V. N. Petrov, and J. H. Dil, "Concept of a multichannel spin-resolving electron analyzer based on Mott scattering," Journal of Synchrotron Radiation 22, 708-716 
(2015).

${ }^{16}$ M. Hoesch, T. Greber, V. N. Petrov, M. Muntwiler, M. Hengsberger, W. Auwaerter, and J. Osterwalder, "Spin-polarized Fermi surface mapping," Journal of Electron Spectroscopy and Related Phenomena 124, 263-279 (2002).

${ }^{17}$ The easiest way to obtain $L_{1}^{*}, L_{2}^{*}$ and $L_{3}^{*}$ is to repeat the same calculation for $L_{4}^{*}$ with labels rotated. The result is expressed by substituting all the labels $(1,2,3,4 *)$ in Eq. (13) with $(2,3,4,1 *),(3,4,1,2 *)$ or $(4,1,2,3 *)$ respectively.

${ }^{18}$ T. Pincelli, F. Grasselli, V. N. Petrov, P. Torelli, and G. Rossi, "Performance of photoelectron spin polarimeters with continuous and pulsed sources: from storage rings to free electron lasers," Journal of Synchrotron Radiation 24, 175-187 (2017).

${ }^{19}$ F. Meier, J. H. Dil, J. Lobo-Checa, L. Patthey, and J. Osterwalder, "Quantitative vectorial spin analysis in angle-resolved photoemission: $\mathrm{Bi} / \mathrm{Ag}(111)$ and $\mathrm{Pb} / \mathrm{Ag}(111)$," Physical Review B 77, 165431 (2008). 
